\title{
MULTIRATE STATE ESTIMATION USING MOVING HORIZON ESTIMATION
}

\author{
Stefan Krämer* and Ralf Gesthuisen* \\ * Deutsche BP AG, Chemicals Production Köln, Postfach 750212, \\ 50754 Köln, stefan.kraemer@de.bp.com
}

\begin{abstract}
In most chemical processes only some measurements are available online while other measurements are available infrequently and often with long delays. Multirate state estimation can optimally combine these different classes of measurements to improve the estimation quality compared to the fast measurements alone. The nature of measurements at different sampling intervals which are subject to delays makes the application of traditional one step state estimators cumbersome. There is but one state estimation scheme which naturally suggests the inclusion of these different classes of measurements, the Moving Horizon Estimator (MHE). In this paper, we extend the MHE concept to the multirate case (MMHE). We present two forms, a variable structure and a fixed structure MMHE and present the relevant equations. We recommend the fixed structure estimator as it has superior noise reductions qualities. The proposed scheme is supported by a simulation example. Copyright (c)2005 IFAC
\end{abstract}

Keywords: multirate state estimation, moving horizon estimation, nonlinear state estimation

\section{INTRODUCTION}

In most chemical processes, only some measurements such as temperatures and flow rates are available online, whereas other variables such as concentrations are usually measured infrequently and possibly with long delays. Often, measurements of the other state variables of the process are not available at all (inner states). For process operation, optimisation and online control it is often desirable to know all states of the system. State or parameter estimation is therefore part of many advanced control and optimisation strategies. In this paper, we extend the nonlinear Moving Horizon Estimator (MHE) to a Multirate MHE (MMHE) as the horizon allows for the inclusion of time delays and naturally suggests the use of measurements at different sampling intervals. We present two forms, a variable structure and a fixed structure MMHE and present the relevant equations. We advocate the use of the fixed structure because it distributes the correction caused by the slow measurements over the slow sampling period, leading to a smoother estimation. Simulation results are given to underline our findings.
Multirate state estimation is related to multirate control, which is well known. Glasson (1983) defines multirate output control, where many outputs are sampled before one control action is performed, and multirate input control, where many control actions are performed to influence one sampled output. The considered state estimation schemes are related to and useful for multirate input control.

An iterated EKF (Jazwinski, 1970) with variable structure for an augmented linearisation of the nonlinear system was developed by Gudi et al. (1995) and applied to a bioreactor. The iterated EKF was used as strong nonlinearities are present in the output equation. Myers et al. (1996) report a similar application, where a variable structure EKF was used and the states were re-estimated from the point in time when the second measurement becomes available. Although simple, the approach results in better estimates as additional information is used. Mutha and Cluett (1997) developed an EKF with fixed lag smoothing, where a variable structure EKF was used. The authors showed that better results could be obtained for an 
example which is not observable from the fast measurements alone.

A different approach based on the concept of the nonlinear reduced observer (Soroush, 1997) was reported by Zambare $\boldsymbol{e t}$ al. (2001). In this estimator, the missing samples of the slow measurement are compensated by a polynomial extrapolation, which in contrast to the model does not react to input changes. This results in a large error in the case of changes of the inputs. Their "illustrative example" clearly shows this problem without it being raised by the authors.

\section{MULTIRATE STATE ESTIMATION}

In the literature, multirate systems are often defined as systems where the measurements and the controls are sampled or applied at different sampling rates. Here we consider the case where in a multivariate system different measurements are available at different sampling rates and with different delays.

For clarity, the derivations are performed for sampled systems with two measurement vectors, one of which is available at a fast sampling rate while the other one is available at a slow sampling rate. A slow sampling point always coincides with a fast sampling point. We label the time between two slow sampling points the intersampling period. The slow sampling time and the time delay are integer multiples of the fast sampling time. Three different situations can be considered, which are depicted in Figure 1.

If $j$ is a step from $t$ to $t+\Delta t$ with $\Delta t$ being the fast sampling interval and $T_{S}$ represents the number of steps between the slow sampling points, the class of systems considered can be written as

$$
\begin{aligned}
\mathbf{x}_{j+1} & =\mathbf{F}\left(\mathbf{x}_{j}, \mathbf{u}_{j}\right) \\
\mathbf{y}_{j} & = \begin{cases}\mathbf{H}^{F}\left(\mathbf{x}_{j}\right) & j / T_{S} \notin \mathbb{N} \\
\mathbf{H}^{F S}\left(\mathbf{x}_{j}, \mathbf{x}_{j-t_{d}}\right) & j / T_{S} \in \mathbb{N}\end{cases}
\end{aligned}
$$

where ${ }^{F}$ indicates fast, ${ }^{S}$ indicates slow and ${ }^{F S}$ indicates the use of both measurements. At $k / T_{S} \in \mathbb{N}$, the separation $\mathbf{y}^{T}=\left(\mathbf{y}^{F}, \mathbf{y}^{S}\right)$ has to be possible.
Traditional multirate state estimation is used for smoothing of control action, i.e. the controller sampling rate is faster than the measurement sampling rate and thus the estimator interpolates between the measurements by setting the error constant for the intersampling points. Such a state estimator without time delay is written as

$$
\hat{\mathbf{x}}_{k+1}=\mathbf{F}\left(\hat{\mathbf{x}}_{k}, \mathbf{u}_{k}\right)+\mathbf{K}\left(\mathbf{y}_{l}-\mathbf{H}\left(\hat{\mathbf{x}}_{l}\right)\right), l=T_{S}\left\lfloor\frac{k}{T_{S}}\right\rfloor
$$

where $\lfloor$.$\rfloor indicates the nearest smaller integer value.$ The alternative way of using the state estimator is to only apply correction action when measurement information is available, i.e.

$$
\begin{aligned}
\hat{\mathbf{x}}_{k+1} & =\mathbf{F}\left(\hat{\mathbf{x}}_{k}, \mathbf{u}_{k}\right)+ \begin{cases}\mathbf{K}\left(\mathbf{y}_{l}-\mathbf{H}\left(\hat{\mathbf{x}}_{l}\right)\right) & l=k \\
\mathbf{0} & l \neq k\end{cases} \\
l & =T_{S} \cdot\left\lfloor\frac{k}{T_{S}}\right\rfloor
\end{aligned}
$$

Estimator (2) reaches a smoother error decay than estimator (3) and is less susceptible to noisy measurements due to the smaller gain needed. However, the system cannot reach the correct steady state at the intersampling points.

This method can be extended to multirate measurements, whereby the time delay is ignored for clarity. Setting $l=T_{S} \cdot\left\lfloor k / T_{S}\right\rfloor$, the variable and fixed structure state estimators can be written as follows:

- Variable structure

$$
\begin{aligned}
\hat{\mathbf{x}}_{k+1}= & \mathbf{F}\left(\hat{\mathbf{x}}_{k}, \mathbf{u}_{k}\right)+ \\
& \begin{cases}\mathbf{K}^{F}\left(\mathbf{y}_{k}-\mathbf{H}^{F}\left(\hat{\mathbf{x}}_{k}\right)\right) & l \neq k \\
\left(\begin{array}{c}
\mathbf{K}^{F}\left(\mathbf{y}_{k}-\mathbf{H}^{F}\left(\hat{\mathbf{x}}_{k}\right)\right) \\
\mathbf{K}^{S}\left(\mathbf{y}_{l}-\mathbf{H}^{S}\left(\hat{\mathbf{x}}_{l}\right)\right)
\end{array}\right) & l=k\end{cases}
\end{aligned}
$$

- Fixed structure

$$
\hat{\mathbf{x}}_{k+1}=\mathbf{F}\left(\hat{\mathbf{x}}_{k}, \mathbf{u}_{k}\right)+\mathbf{K}\left(\begin{array}{c}
\mathbf{y}_{k}^{F}-\mathbf{H}^{F}\left(\hat{\mathbf{x}}_{k}\right) \\
\mathbf{y}_{l}^{S}-\mathbf{H}^{S}\left(\hat{\mathbf{x}}_{l}\right)
\end{array}\right)
$$

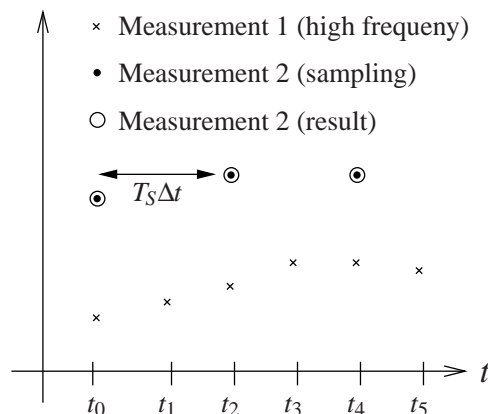

(a) Measurement 2 is available at longer sampling intervals

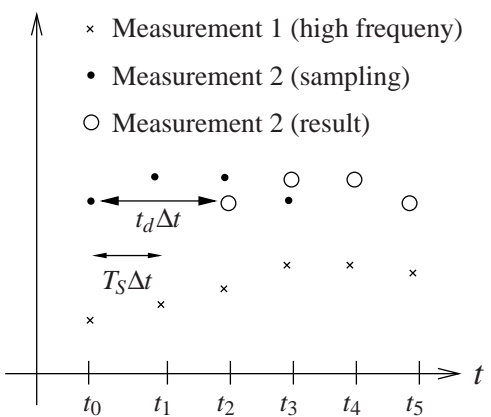

(b) Measurement 2 is available with a delay at the same sampling frequency as the fast measurement

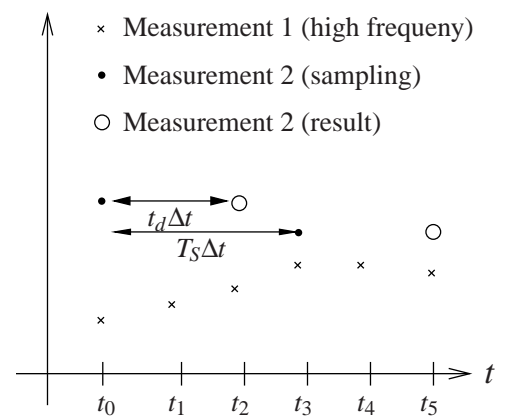

(c) Measurement 2 is available with a delay and at longer sampling intervals

Fig. 1. The different measurement cases $\left(T_{S}\right.$ - intersampling rate, $t_{d}$ - time delay, $\Delta t-$ sampling time $)$ 


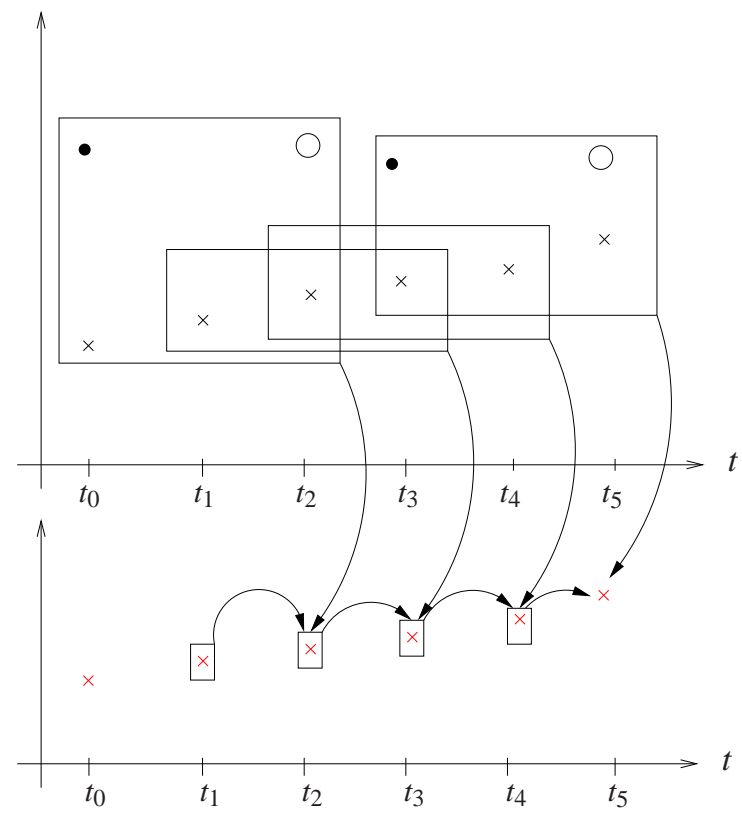

Fig. 2. Multirate MHE (MMHE): Lower co-ordinates are the estimates, upper co-ordinates are the used measurements, horizon is framed. (.) is the sampling point, $(\circ)$ is the time when the slow measurement becomes available and $(\times)$ is the sampling of the fast measurement.

\section{MULTIRATE MOVING HORIZON ESTIMATOR}

A Kalman Filter can be formulated as an optimal state regulator problem and the covariance matrices can be generally considered as weighting matrices of the estimation errors. The solution to this optimisation problem is given by the Matrix-Riccati-Equation (Jazwinski, 1970). Due to the increase in computational speed and improvements in software for solving optimisation problems, optimisation based approaches have been developed in the last decades (Jang et al., 1986; Muske et al., 1993). Here, the calculation of the correction part of the EKF is replaced by an optimisation problem. The major advantage is the capability of incorporating constraints and thus forcing the estimator into a physically sensible solution using additional process knowledge. Imposing constraints is especially important for nonlinear estimation as the estimator may converge to a physically wrong solution (Gesthuisen et al., 2001). Such an estimator is called the Constrained Extended Kalman Filter (CEKF).

For the Moving Horizon Estimator formulated by Muske et al. (Muske et al., 1993) the concept of the $\mathrm{CEKF}$ is extended over a given horizon. The development of the MHE therefore follows naturally from a combination of the Batch Least Squares Estimator (Jazwinski, 1970) and the recursive nature of the Extended Kalman Filter. Its major advantage is that within the horizon all process information is used and the nonlinear model is not linearised. In the BLS, every new measurement increases the size of the optimisation problem, which is why for practical application the size of the optimisation problem has to be limited. Muske et al. (1993) developed a recursive formulation on a moving horizon. According to the notation of Muske et al., the horizon length is $N$ and the number of used measurements $N+1$. $\hat{\mathbf{x}}_{j \mid k}$ is the estimation of the state vector at time $t=t_{j}$ using measurements up to $t=t_{k} . \mathbf{P}_{k-N}, \mathbf{Q}$ and $\mathbf{R}$ are weighting matrices of the estimation, model and measurement error, respectively. Muske et al. use the same covariance matrices as in a similar EKF.

The Multirate Moving Horizon Estimator (MMHE) uses the concept of the MHE, i.e. the incorporation of past measurements, to cope with multirate measurements and time delays without the need for an augmented system or re-iteration. Figure 2 shows the way this is done: When the time delay is passed, the slow measurement is included in the horizon at the sampling point. This is the reason why the horizon length $N$ should always be at least $t_{d}$.

The formulation of the MMHE is based on a nonlinear model of the form

$$
\begin{aligned}
\mathbf{x}_{k+1} & =\mathbf{F}\left(\mathbf{x}_{k}, \mathbf{u}_{k}\right)+\xi_{k} \\
\mathbf{y}_{k} & = \begin{cases}\mathbf{H}^{F}\left(\mathbf{x}_{k}\right)+\varphi_{k}^{F} & k / T_{S} \notin \mathbb{N} \\
\mathbf{H}^{F S}\left(\mathbf{x}_{k}, \mathbf{x}_{k-t_{d}}\right)+\varphi_{k}^{F S} & k / T_{S} \in \mathbb{N}\end{cases} \\
\text { with } \quad \varphi^{F S} & =\left(\varphi^{F} \varphi^{S}\right)^{T} .
\end{aligned}
$$

The MMHE directly derived from the MHE results in a variable structure form. This is the one shown in Figure 2 as only the measurements, which are really available, are used in the horizon. Under the viable assumption

$$
\mathbf{R}_{\mathrm{var}}^{F S}=\left(\begin{array}{cc}
\mathbf{R}^{F} & \mathbf{0} \\
\mathbf{0} & \mathbf{R}_{\mathrm{var}}^{S}
\end{array}\right), \quad \mathbf{R}_{\mathrm{fixed}}=\left(\begin{array}{cc}
\mathbf{R}^{F} & \mathbf{0} \\
\mathbf{0} & \mathbf{R}_{\mathrm{fixed}}^{S}
\end{array}\right)
$$

its formulation is given as follows:

$$
\begin{aligned}
\min _{\hat{\xi}, \hat{\varphi}^{F}, \hat{\varphi}^{S}} & \Psi_{k}= \\
& \hat{\xi}_{k-N-1 \mid k}^{T} \mathbf{P}_{k-N \mid k-1}^{-1} \hat{\xi}_{k-N-1 \mid k} \\
& +\sum_{j=k-N}^{k-1} \hat{\xi}_{j \mid k}^{T} \mathbf{Q}^{-1} \hat{\xi}_{j \mid k} \\
& +\sum_{j=k-N}^{k}\left(\hat{\varphi}_{j \mid k}^{F}\right)^{T}\left(\mathbf{R}^{F}\right)^{-1} \hat{\varphi}_{j \mid k}^{F} \\
& +\sum_{\substack{j=k-N \\
j-t_{d}-k-N}}^{k}\left(\hat{\varphi}_{j \mid k}^{S}\right)^{T}\left(\mathbf{R}_{\mathrm{var}}^{S}\right)^{-1} \hat{\varphi}_{j \mid k}^{S} \in \mathbb{N} \\
\text { s.t. } \quad \hat{\mathbf{x}}_{k-N \mid k}= & \hat{\mathbf{x}}_{k-N \mid k-1}+\hat{\xi}_{k-N-1 \mid k} \\
\hat{\mathbf{x}}_{j+1 \mid k}= & \mathbf{F}\left(\hat{\mathbf{x}}_{j \mid k}, u_{j}\right)+\hat{\xi}_{j \mid k} \\
& j=k-N, \ldots, k-1
\end{aligned}
$$




$$
\begin{gathered}
\hat{\varphi}_{j \mid k}^{F}=\mathbf{y}_{j}-\mathbf{H}^{F}\left(\hat{\mathbf{x}}_{j \mid k}\right) \\
j=k-N, \ldots, k \wedge j / T_{S} \notin \mathbb{N} \\
\left(\begin{array}{c}
\hat{\varphi}_{j \mid k}^{F} \\
\hat{\varphi}_{j \mid k}^{S}
\end{array}\right)=\mathbf{y}_{j}-\mathbf{H}^{F S}\left(\hat{\mathbf{x}}_{j \mid k}, \hat{\mathbf{x}}_{j-t_{d} \mid k}\right) \\
j / T_{S} \in \mathbb{N} \wedge j-t_{d} \geq k-N \\
\xi_{\min } \leq \hat{\xi}_{j-1 \mid k} \leq \xi_{\max } \\
\varphi_{\min }^{F} \leq \hat{\varphi}_{j \mid k}^{F} \leq \varphi_{\max }^{F} \\
\varphi_{\min }^{S} \leq \hat{\varphi}_{j \mid k}^{S} \leq \varphi_{\max }^{S} \\
\mathbf{x}_{\min } \leq \hat{\mathbf{x}}_{j \mid k} \leq \mathbf{x}_{\max }
\end{gathered}
$$

It has to be noted that in contrast to the original approach proposed in (Muske et al., 1993) the initial state vector at the beginning of the horizon is based on the measurements up to time $t=t_{k-1}$, i.e. it is a smoothed vector instead of a predicted one $\left(\hat{\mathbf{x}}_{k-N \mid k-1}\right.$ instead of $\hat{\mathbf{x}}_{k-N \mid k-N-1}$ ). This formulation of the MHE is proposed by Tenny (2002) and improves the performance of the estimations. The prediction also has to be used in the variable structure.

$$
\begin{aligned}
\hat{\mathbf{x}}_{i+1 \mid k-1} & =\mathbf{F}\left(\hat{\mathbf{x}}_{i \mid k-1}, \mathbf{u}_{i}\right) \\
\mathbf{P}_{i+1 \mid k-1} & =\mathbf{A}_{i \mid k-1}\left(\mathbf{P}_{i \mid k-2}-\mathbf{P}_{i \mid k-2} \mathbf{H}_{i \mid k-1}^{T}\right.
\end{aligned}
$$

$$
\begin{aligned}
& \left(\mathbf{H}_{i \mid k-1} \mathbf{P}_{i \mid k-2} \mathbf{H}_{i \mid k-1}^{T}+\mathbf{R}\right)^{-1} \\
& \left.\mathbf{H}_{i \mid k-1} \mathbf{P}_{i \mid k-2}\right) \mathbf{A}_{i \mid k-1}^{T}+\mathbf{Q}
\end{aligned}
$$

with

$$
\mathbf{H}_{i \mid k-1}= \begin{cases}\partial \mathbf{H}^{F}(\mathbf{x}) /\left.\partial \mathbf{x}\right|_{\hat{\mathbf{x}}_{i \mid k-1}} & i / T_{S} \notin \mathbb{N} \\ \partial \mathbf{H}^{F S}(\mathbf{x}) /\left.\partial \mathbf{x}\right|_{\hat{\mathbf{x}}_{i \mid k-1}} & i / T_{S} \in \mathbb{N}\end{cases}
$$$$
\mathbf{R}=\left\{\begin{array}{ll}
\mathbf{R}^{F} & i / T_{S} \notin \mathbb{N} \\
\mathbf{R}^{F S} & i / T_{S} \in \mathbb{N}
\end{array},\right.
$$

and

$$
\begin{aligned}
\mathbf{A}_{i \mid k-1} & =\partial \mathbf{f}(\mathbf{x}) /\left.\partial \mathbf{x}\right|_{\hat{\mathbf{x}}_{i \mid k-1}} \\
i & =k-N-1
\end{aligned}
$$

Unfortunately, this variable structure results in step wise improvement of the estimation. As $\mathbf{P}$ can change dramatically when more measurements are available, it is also believed that cycling behaviour can occur. Figure 3 shows this behaviour for the estimation of the leakage in a linearised three tank system. While for some systems this stepping behaviour is an advantage, for most systems using the estimation for multirate input control, the smooth error decay of a fixed structure state estimator, as also shown in the figure, is the preferable choice.

This fixed structure places the last error $\left(\mathbf{y}_{l}-\hat{\mathbf{y}}_{l}\right)$ of the slow measurement on a zero order hold. This has to be done beyond the sampling point of the next measurement, as it is again subject to the delay. At the moment when the new measurement becomes available, the former errors between the corresponding sampling point and the current time in the horizon have to be replaced by the new value of the error. The fixed structure MMHE formulation is given by

$$
\begin{aligned}
\min _{\hat{\xi}, \hat{\varphi}} & \Psi_{k}=\hat{\xi}_{k-N-1 \mid k}^{T} \mathbf{P}_{k-N \mid k-1}^{-1} \hat{\xi}_{k-N-1 \mid k} \\
& +\sum_{j=k-N}^{k-1} \hat{\xi}_{j \mid k}^{T} \mathbf{Q}^{-1} \hat{\xi}_{j \mid k} \\
& +\sum_{j=k-N}^{k} \hat{\varphi}_{j \mid k}^{T} \mathbf{R}_{\text {fixed }}^{-1} \hat{\varphi}_{j \mid k} \\
\text { s.t. } \quad \hat{\mathbf{x}}_{k-N \mid k}= & \hat{\mathbf{x}}_{k-N \mid k-1}+\hat{\xi}_{k-N-1 \mid k} \\
\hat{\mathbf{x}}_{j+1 \mid k}= & \mathbf{F}\left(\hat{\mathbf{x}}_{j \mid k}, u_{j}\right)+\hat{\xi}_{j \mid k} \\
& j=k-N, \ldots, k-1
\end{aligned}
$$

$$
\begin{gathered}
\hat{\varphi}_{j \mid k}=\left(\begin{array}{c}
\mathbf{y}_{j}^{F}-\mathbf{H}^{F}\left(\hat{\mathbf{x}}_{j \mid k}\right) \\
\mathbf{y}_{l}^{S}-\mathbf{H}^{S}\left(\hat{\mathbf{x}}_{l \mid \bar{k}}\right)
\end{array}\right) \\
j=k-N, \ldots, k \\
l=\left\{\begin{array}{c}
T_{S}\left(\left\lfloor j / T_{S}\right\rfloor-1\right) \\
\forall\left(j \geq T_{S}\left\lfloor k / T_{S}\right\rfloor\right) \\
\wedge\left(k<T_{S}\left\lfloor k / T_{S}\right\rfloor+t_{d}\right) \\
T_{S}\left\lfloor j / T_{S}\right\rfloor \quad \text { otherwise }
\end{array}\right. \\
\bar{k}=\left\{\begin{array}{c}
k \quad \forall l \leq k-N \\
l+N \quad \text { otherwise }
\end{array}\right. \\
\xi_{\min } \leq \hat{\xi}_{j-1 \mid k} \leq \xi_{\max } \\
\varphi_{\min } \leq \hat{\varphi}_{j \mid k} \leq \varphi_{\max } \\
\mathbf{x}_{\min } \leq \hat{\mathbf{x}}_{j \mid k} \leq \mathbf{x}_{\max }
\end{gathered}
$$

$\bar{k}$ is necessary if $l$ lies outside the horizon. The prediction of $\mathbf{P}$ remains the same as if all measurements were always available.

$$
\begin{aligned}
\hat{\mathbf{x}}_{i+1 \mid k-1}= & \mathbf{F}\left(\hat{\mathbf{x}}_{i \mid k-1}, \mathbf{u}_{i}\right) \\
\mathbf{P}_{i+1 \mid k-1}= & \mathbf{A}_{i \mid k-1}\left(\mathbf{P}_{i \mid k-2}-\mathbf{P}_{i \mid k-2} \mathbf{H}_{i \mid k-1}^{T}\right. \\
& \left(\mathbf{H}_{i \mid k-1} \mathbf{P}_{i \mid k-2} \mathbf{H}_{i \mid k-1}^{T}+\mathbf{R}_{\text {fixed }}\right)^{-1} \\
& \left.\mathbf{H}_{i \mid k-1} \mathbf{P}_{i \mid k-2}\right) \mathbf{A}_{i \mid k-1}^{T}+\mathbf{Q}
\end{aligned}
$$

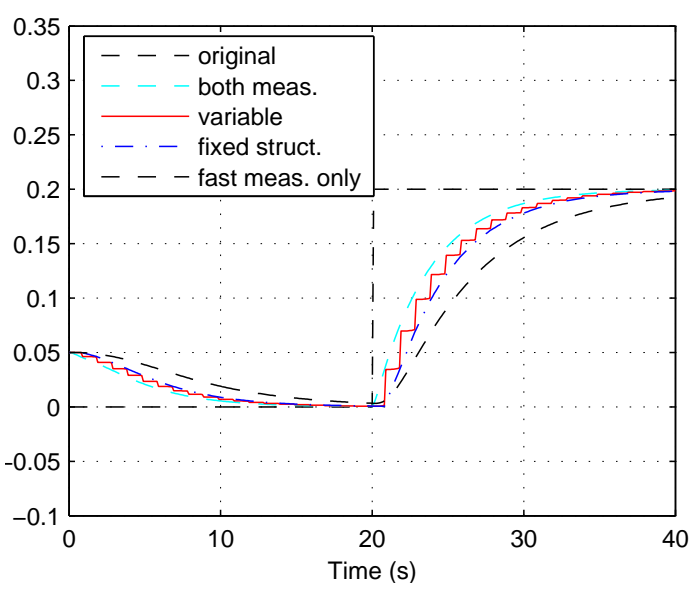

Fig. 3. Linear MMHE for the leakage in a three tank system: Cautious tuning, $T_{S}=10, t_{d}=0 \mathrm{~s}$. 

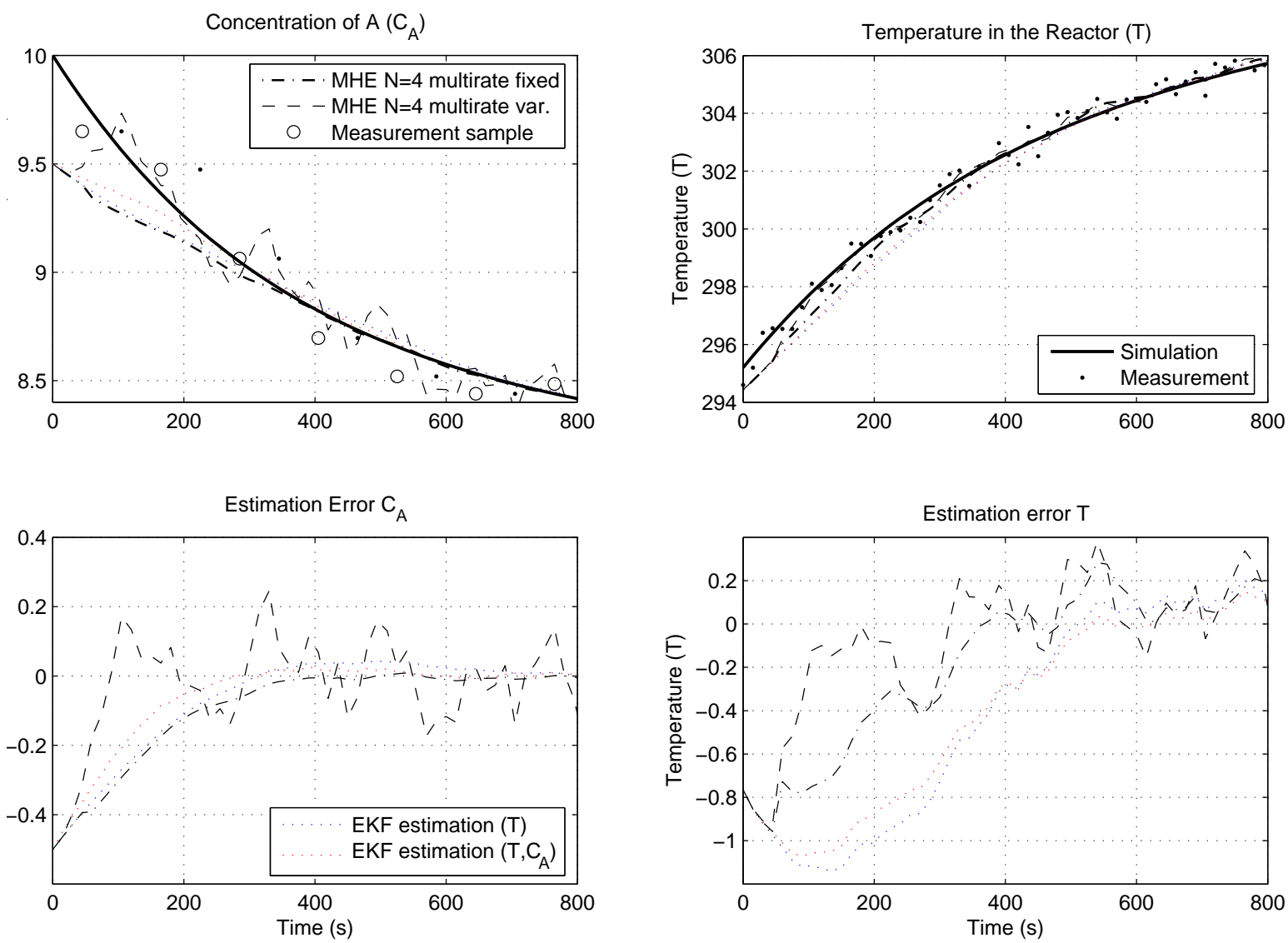

Fig. 4. MMHE estimate of the concentration of A and the temperature in the CSTR example - noisy measurements

$$
\text { with } \quad \begin{array}{rlrl} 
& \mathbf{H}_{i \mid k-1} & =\left.\frac{\partial \mathbf{h}(\mathbf{x})}{\partial \mathbf{x}}\right|_{\hat{\mathbf{x}}_{i \mid k-1}} \\
& & \mathbf{A}_{i \mid k-1} & =\left.\frac{\partial \mathbf{f}(\mathbf{x})}{\partial \mathbf{x}}\right|_{\hat{\mathbf{x}}_{i \mid k-1}} \\
\text { and } & & i & =k-N-1
\end{array}
$$

The above equations are written for a regular slow measurement. Implementation in a computer system does not require this regularity.

The purpose of putting the unavailable measurement on zero order hold is to spread the required amplification of the estimator along the frequent measurements. It should be guaranteed that the estimator does not interpret this error as a new error but uses the required fractional amplification. One is inclined to think this can be achieved by fixing the slow measurement part of $\hat{\varphi}$ when no fast measurement is available. The formulation in (12) already does this as $\mathbf{y}_{l}^{S}-\mathbf{H}^{S}\left(\hat{\mathbf{x}}_{l \mid k}\right)$ is constant between the different $l$. Therefore, the whole $\hat{\xi}_{j \mid k}$ depends on the spread amplification of the slow measurement and the current frequent measurement.

In order to be comparable with the variable structure, the measurement covariance $\mathbf{R}^{S}$ of the slow measurements has to be adjusted.

If $v_{i}$ are $n$ zero mean estimators (e.g. measurements) and variance $\sigma_{i}^{2}$, the optimal combination of these estimators $v$ has the variance

$$
\sigma^{2}=\frac{\prod_{i}^{n} \sigma_{i}^{2}}{\sum_{i}^{n} \prod_{j \neq i}^{n} \sigma_{j}^{2}} .
$$

If $\sigma_{i}$ are the same, i.e. $\bar{\sigma}^{2}=\sigma_{1}^{2}=\cdots=\sigma_{n}^{2}$,

$$
\sigma^{2}=\frac{\bar{\sigma}^{2}}{n} \text {. }
$$

If for the fixed structure MMHE we assume one estimated error is not taken at one point with variance $\mathbf{R}_{\mathrm{var}}^{S}$ but at a number of $T_{S}$ equally important points, we can say

$$
\left(\begin{array}{cc}
\mathbf{R}^{F} & \mathbf{0} \\
\mathbf{0} & T_{S} \mathbf{R}_{\mathrm{var}}^{S}
\end{array}\right)=\left(\begin{array}{cc}
\mathbf{R}^{F} & \mathbf{0} \\
\mathbf{0} & \mathbf{R}_{\text {fixed }}^{S}
\end{array}\right) .
$$

Using this relationship, equivalent tuning of the two structures can be obtained as was used in Figures 3 and 4.

\section{SIMULATIVE EXAMPLE}

In order to compare the performance of the different approaches developed in this paper to the usually applied EKF, the different methods are applied to a nonlinear example process taken from the literature.

Example 1. (Soroush and Kravaris (1992)). A CSTR wherein the irreversible reactions 


$$
\begin{array}{ll}
A \stackrel{k_{1}}{\longrightarrow} U_{1} & r_{A}^{1}=-k_{1} C_{A}^{3} \\
A \stackrel{k_{2}}{\longrightarrow} U_{2} & r_{A}^{1}=-k_{2} C_{A}^{\frac{1}{2}} \\
A \stackrel{k_{d}}{\longrightarrow} U_{D} & r_{A}^{1}=-k_{d} C_{A}
\end{array}
$$

take place. The following observable differential equations are considered:

$$
\begin{aligned}
\frac{d C_{A}}{d t} & =R_{A}\left(C_{A}, T\right)+\frac{\dot{V}}{V_{R}}\left(C_{A, i n}-C_{A}\right) \\
\frac{d T}{d t} & =\frac{R_{H}}{\rho c_{p}}+\frac{\dot{V}}{V_{R}}\left(T_{i n}-T\right)+\frac{\dot{Q}}{\rho c_{p} V_{R}}
\end{aligned}
$$

where $R_{A}\left(C_{A}, T\right)=-k_{1}(T) C_{A}^{3}-k_{2}(T) C_{A}^{\frac{1}{2}}-k_{d}(T) C_{A}$

$$
\begin{aligned}
& k_{1}(T)=k_{1}^{0} e^{-\frac{E_{A, 1}}{R T}} \\
& k_{2}(T)=k_{2}^{0} e^{-\frac{E_{A, 2}}{R T}} \\
& k_{d}(T)=k_{d}^{0} e^{-\frac{E_{A, d}}{R T}}
\end{aligned}
$$

The inputs are $\left(C_{A, i n}, T_{i n}, Q\right)^{T}$, however, $C_{A, \text { in }}$ is the only manipulated variable. For $\dot{Q}=-1.030 \mathrm{~kW}$ this CSTR exhibits three steady states. The operating conditions, steady states and physical properties are used as in Soroush and Kravaris (1992).

In Figure 4 the estimators are given noisy measurements. Only the MMHE receives multirate measurements with measurements delays, the measurements given to the EKF are single rate measurements without delay. The fixed structure MMHE converges onto the real value smoothly. Despite the EKF looking better than the fixed structure MMHE, the MMHE reaches zero error at around $400 \mathrm{~s}$ whereas the EKF with one measurement (only temperature) needs up until $650 \mathrm{~s}$. The advantage of the fixed structure MMHE is especially obvious in the noisy case. The noise is strongly amplified by the variable structure MMHE, whereas the fixed structure MMHE, just as the EKF, remains smooth. It has to be noted that the fixed structure MMHE performs similar to an EKF which at every sampling point takes into account both the temperature and the concentration measurement.

\section{CONCLUSION}

In this paper we investigate the use of multirate delayed measurements in state estimation schemes. We consider the Moving Horizon Estimator the best starting point for the combination of the different classes of measurements into one state estimation scheme and extend the MHE to a Multirate MHE, which can handle the different measurement. We discuss a variable and a fixed structure and recommend the fixed structure, as it distributes the amplification of the error between the predicted and the measured slow measurements over several fast measurements between two slow samples and results in a smoother decay of the error. A simulative example of a CSTR underlines the proposed approach.

\section{ACKNOWLEDGEMENTS}

We gratefully acknowledge the fruitful discussions with Prof. Sebastian Engell, Chair of Process Control, Department of Chemical and Biochemical Engineering, Universität Dortmund. Parts of this work were funded by the DFG (Deutsche-ForschungsGemeinschaft), DFG-Project EN 152/31-1. We gratefully acknowledge this financial support.

\section{REFERENCES}

Gesthuisen, Ralf, Stefan Krämer and Sebastian Engell (2001). State estimation for semicontinuous emulsion copolymerization using calorimetric data. In: Proceedings of the American Control Conference (Bruce H. Krogh, Ed.). American Automatic Control Council. Arlington, Virginia. pp. 362-367.

Glasson, Douglas P. (1983). Development and application of multirate digital control. Control Systems Magazine pp. 2-8.

Gudi, Ravindra D., Sirish L. Shah and Murray R. Gray (1995). Adaptive multirate state and parameter estimation strategies with application to a bioreactor. AIChE Journal 41(11), 2451-2464.

Jang, Shi-Shang, Babu Joseph and Hiro Mukai (1986). Comparison of two approaches to on-line parameter and state estimation of nonlinear systems. Ind. Eng. Chem. Process Des. Dev. 25, 809-814.

Jazwinski, A.H. (1970). Stochastic Processes and Filtering Theory. Academic Press, New York.

Muske, K. R., J. B. Rawlings and J. H. Lee (1993). Receding horizon recursive state estimation. Proceedings of the American Control Conference, San Francisco pp. 900-904.

Mutha, Rajenda K. and William R. Cluett (1997). On-line nonlinear model-based estimation and control of a polymer reactor. AIChE Journal 43(11), 3042-3058.

Myers, M. A., S. Kang and R. H. Luecke (1996). State estimation and control for systems with delayed off-line measurements. comp. chem. eng. 20(5), 585-588.

Soroush, M. and C. Kravaris (1992). Discrete-time nonlinear controller synthesis by input/output linearization. AIChE Journal 40, 980-992.

Soroush, Masoud (1997). Nonlinear state-observer design with application to reactors. Chemical Engineering Science 52(3), 387-404.

Tenny, Matthew J. (2002). Computational Strategies for Nonlinear Model Predictive Control. PhD thesis. University of Wisconsin-Madison.

Zambare, Neeraj, Masoud Soroush and Babatunde A. Ogunnaike (2001). Robustness improvement in multi-rate state estimation. In: Proceedings of the American Control Conference (Bruce H. Krogh, Ed.). American Automatic Control Council. Arlington, Virginia. pp. 993-998. 